Conclusion Results of this study showed, that hypertensive adolescents have poorer oral health than adolescents with normal BP level. This substantiates the necessity of multidisciplinary approach to management of this group of patients with participation of both pediatric physicians and dentists.

\section{P60 CLINICAL CHARACTERISTICS, COMPLICATIONS, AND ANTICOAGULATION IN CHILDREN WITH INFECTIVE ENDOCARDITIS: A SINGLE CENTER EXPERIENCE FROM TURKEY}

${ }^{1}$ Pelin Kosger, ${ }^{2}$ Tugcem Keskin, ${ }^{2}$ Hikmet Kiztanir, ${ }^{3}$ Birgul Kirel, ${ }^{2}$ Birsen Ucar*. ${ }^{*}$ Eskisehir Osmangazi Universty, Faculty of Medicine, department of Pediatric Cardiology, Eskisehir, Turkey; ${ }^{2}$ Eskisehir Osmangazi Universty, Faculty of Medicine, department of Pediatric Cardiology, Eskisehir, Turkey; ${ }^{3}$ Eskisehir Osmangazi Universty, Faculty of Medicine, department of Pediatric Endocrinology, Eskisehir, Turkey

10.1136/archdischild-2019-epa.415

Objective In this study, the data of our patients diagnosed with infective endocarditis (IE) between 2010-2018 at the Eskişehir Osmangazi University Faculty of Medicine Hospital, Department of Pediatric Cardiology were retrospectively obtained.

Methods Demographic data, the presence of a predisposing cardiac disease, presentation with complaints, clinical and laboratory findings, blood culture results, treatment plans, evolving complications, and the echocardiographic data of eleven patients were examined. The relevant features of the patients with complications, of those having anticoagulant therapy in addition to antibiotherapy, those who underwent surgery, and those who showed a mortality course were determined.

Results The youngest patient was 7 months old and the oldest was 14 years old $(7.5 \pm 4.6$ years $)$. All of the cases had congenital heart anomalies: four had cyanotic congenital heart disease (CHD), four had a ventricular septal defect (VSD), two had a bicuspid aortic valve (BAV), and one patient had a secundum atrial septal defect (ASD). There were no cases with rheumatic heart disease. Three patients with cyanotic CHD were treated with corrective cardiac surgery. A total of eight patients had embolic findings; five at the time of admission and three at follow-up. Nine patients received antibiotics prior to admission. Echocardiography showed vegetation in nine patients. Anticoagulant treatment was applied to 2 patients with mitral valve and aortic valve vegetation in addition to antibiotic treatment. The most common microorganism in the blood culture was coagulase negative staphylococcus with 5 cases and 2 of them were identified as staph. epidermidis. The second most common microorganism was the viridans group streptococcus with 4 cases. Four patients underwent early surgical treatment, one patient died due to multiple organ failure caused by systemic embolization, and one patient died due to sudden hemodynamic instability in the first week of follow-up.

Conclusion IE is a serious disease with life-threatening complications, where the number of at-risk patients due to $\mathrm{CHD}$ is gradually increasing and thus the mortality rate has not decreased despite improved medical and surgical treatment procedures. Early initiation of appropriate antibiotic treatment following early diagnosis is has great importance in preventing mortality.Considering the complications that may be caused by early surgical intervention, anticoagulant therapy, which is combined with antibiotherapy in appropriate patients, can be tried as a useful option prior to committing to early surgery.

\section{P61 ATRIOVENTRICULAR BLOCK IN CHILDREN - SOMETIME IS A CHALLENGE}

${ }^{1,2}$ Gabriela Doros, ${ }^{1,2}$ Andrada Mara Ardelean* ${ }^{1,2}$ Anca Popoiu, ${ }^{1,2}$ Cristina Olariu ${ }^{1,2}$ Ramona Stroescu, 1,2 Mihai Gafencu. 'Victor Babes University of Medicine and Pharmacy, Timisoara, Romania; ${ }^{2}$ IIIrd Pediatric Clinic Emergency Hospital for Children Louis Turcanu, Timisoara, Romania

\subsection{6/archdischild-2019-epa.416}

Introduction Atrioventricular(AV) blocks in children may become a challenge in some situations and the aim is to present three different cases admitted in our Department.

Material and method A 12 yo girl was admitted for precordial pain during a respiratory infection. An 8 yo boy was admitted for a syncope in the time he was laying in bed near his father. The third case was a 14 yo girl, known from the age of 2,6 yo with gr. III AV block, implanted with an abdominal pace maker(PM) with epicardial leads, changed in 2016 with a bicameral PM, programmed DDD with $60 \mathrm{~b} / \mathrm{min}$; she was admitted for intense fatigue. All of them performed cardiac and arrhythmology examination.

Results The 12 yo girl presented on ECG, grade I AV block, with a PQ interval of $0.30 \mathrm{sec}$. Holter ECG revealed gr I AV block with different PQ intervals, the maximum length being 0.37 sec. and paroxysmal Gr II Mobitz I Block during night. Being asymptomatic, the decision was just to be followed at 6 mo. The boy with the syncope presented on Holter ECG, Gr II Mobitz I AV block during night, that may be a normal condition in asymptomatic children. Both patients had structurally normal heart and function. The third patient presented tachycardia $122 \mathrm{~b} / \mathrm{min}$ and weakness. She had cardiac dissynchrony, EF 53\%, gr I mitral regurgitation, ASD and reduced GLS. The PM was changed to VVI with $50 \mathrm{~b} / \mathrm{min}$. She felt better, but dizzy. Atrial conduction was 120/min and Ventricular spike were at $50 / \mathrm{min}$. Next day PM was upgraded to VVIR, with atrial conduction at $120 / \mathrm{min}$ and ventricular activity at $73 \mathrm{~b} /$ min. Ecogocardiography, EF improved and dissyncrony disappeared. The patient felt much better, being able to be discharged.

Conclussions Gr I AV block, when prolonged PQ interval more than $0.30 \mathrm{sec}$, needs to be investigated. Asymptomatic paroxistic gr II Mobits I block during sleep, has to be monitored. The new concept of PM Syndrome has to be checked and fixed to be safe for the patient. Simple cases in different situations become a challenge for the pediatric cardiology doctor.

\section{P62 A REVIEW OF NEAR INFRA-RED SPECTROSCOPY}

Peter Donnelly*, Miriam Fine-Goulden. Evelina London Children's Hospital, London, UK

10.1136/archdischild-2019-epa.417

Near infra-red spectroscopy (NIRS) is an exciting non-invasive, portable, continuous modality used to assess regional tissue oxygen saturations, expressed as a percentage. The use of NIRS in clinical practice is relatively recent, with its role in measuring cerebral oxygenation originally described in the 1970s.

The primary application of NIRS in paediatrics is in monitoring for signs of reduced or differential tissue perfusion, particularly post cardiac surgery. 